\title{
The Formation of Multiple Milia Induced by Pneumatic Injector Therapy for Scar Remodeling
}

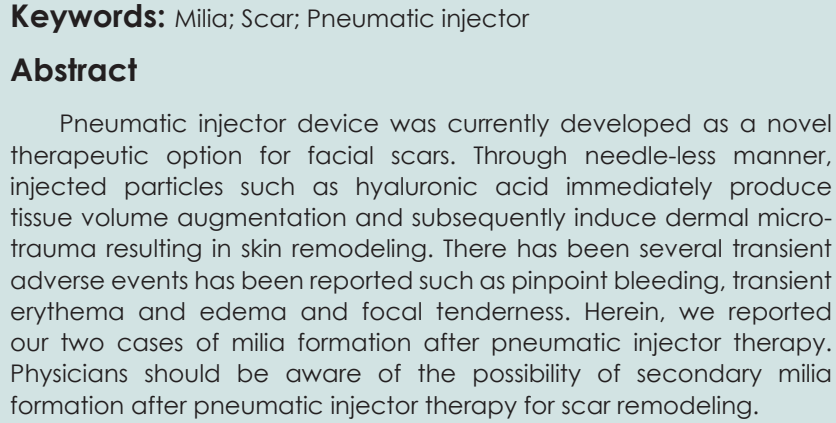

Pneumatic injector device was currently developed as a nove therapeutic option for facial scars. Through needle-less manner, injected particles such as hyaluronic acid immediately produce tissue volume augmentation and subsequently induce dermal microtrauma resulting in skin remodeling. There has been several transient adverse events has been reported such as pinpoint bleeding, transient erythema and edema and focal tenderness. Herein, we reported our two cases of milia formation after pneumatic injector therapy. Physicians should be aware of the possibility of secondary milia formation after pneumatic injector therapy for scar remodeling.

\section{Case Report}

There are various treatment modalities for scar revision, including intralesional steroid injection, ablative laser therapy, cryosurgery, dermabrasion, excisional surgery and subcision. Recently a pneumatic injector device was developed as a novel therapeutic option for facial scars [1]. It accelerates and laterally disperses Hyaluronic Acid (HA) via tiny entry points in the epidermis using pneumatic needle-less action [2]. The HA particles immediately produce tissue volume augmentation and subsequently induce dermal micro-trauma. This process mechanically stretches the fibroblasts, stimulates growth factor secretion and promotes the formation of new collagen fibers, resulting in skin remodeling and improvement in scar appearance [3] A 76-year-old Asian female presented with a linear depressed scar on her chin (Figure 1a). The patient underwent four pneumatic injection treatments with the INNOJECTOR ${ }^{\mathrm{TM}}$ device (Amore Pacific, Seoul, Korea) at four-week intervals. It produces a high-velocity jet (up to

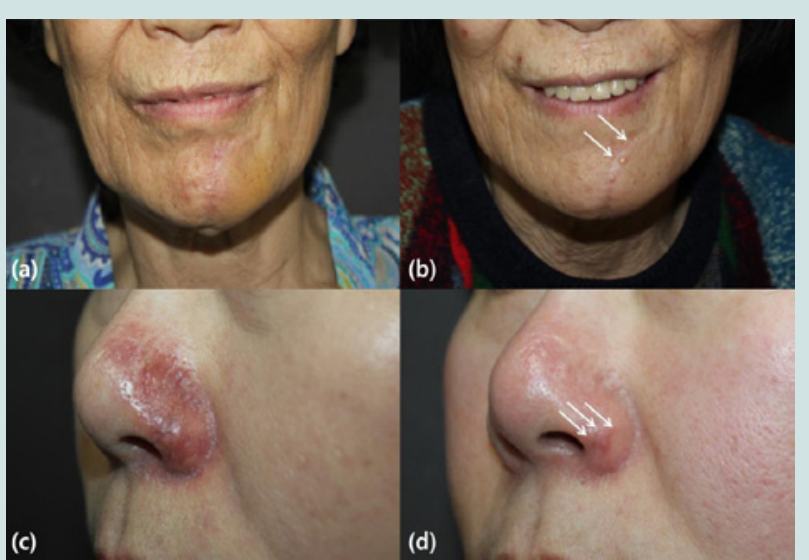

Figure 1: (a) Linear depressed scar on the chin. (b) Improvement of the scar and two milia that developed at the pneumatic injection site after three treatments. (c) Erythematous depressed patches on the left nasal ala. (d) Three tiny milia formed at the scar site treated by pneumatic injection.

\section{Journal of}

\section{Clinical \& Investigative Dermatology}

\section{Sun Young Choi ${ }^{1}$, Ga Ram Ahn², Beom Joon Kim ${ }^{2}$,} Myeung Nam Kim² and Chang Kwun Hong ${ }^{2}$

${ }^{\prime}$ Department of Dermatology, Inje University College of Medicine, Seoul Paik Hospital, South Korea

2Department of Dermatology, Chung-Ang University College of Medicine, South Korea

\section{*Address for Correspondence}

Beom Joon Kim, Department of Dermatology, Chung Ang University Hospital 224-1 Heukseok-dong, Dongjak-ku, Seoul 156-755, South Korea, Tel: 82-2-6299-1525; Fax: 82-2-823-104; E-mail: beomjoon@unitel.co.kr

\section{Submission: 12 October, 2017}

Accepted: 20, November, 2017

Published: 27, November, 2017

Copyright: @ 2017 Choi SY, et al. This is an open access article distributed under the Creative Commons Attribution License, which permits unrestricted use, distribution, and reproduction in any medium, provided the original work is properly cited.

$180 \mathrm{~m} / \mathrm{s}$ ) with a nozzle diameter of $0.1 \mathrm{~mm}$ that penetrates the skin and delivers the solution intradermally using a liquid propelled by compressed gasses (nozzle diameter $0.1 \mathrm{~mm}$, max velocity $180 \mathrm{~m} / \mathrm{s}$ ). Cross-linked biphasic HA (Cleviel volume; Amore Pacific, Seoul, Korea) solution was delivered in a linear distribution along the scar line at $5 \mathrm{~mm}$ intervals and approximately $1 \mathrm{mg} \mathrm{HA}$ was deposited in the treatment area. One week after the final three treatments the appearance of the scar had improved but two milia had developed on the treated site after pneumatic injection (Figure 1b).

A 53-year-old Asian female presented with erythematous depressed patches on the left nasal ala (Figure 1c). She suffered skin necrosis due to arterial obstruction in the area as a complication of the HA filler. The patient received three pneumatic injection treatments with the INNOJECTOR ${ }^{\mathrm{TM}}$ device combined with LED (SMARTLUX, MEDMIX Corporation, Korea) at three-week intervals. We injected HA (Cleviel volume, Amore Pacific, Seoul, Korea) in each jet was $0.15 \mathrm{ml}$ and jets were ejected at a distance of $3 \mathrm{~mm}$. After four weeks of twice weekly treatments the erythema and depression of the scar were improved. However after one week of three treatments per week she presented with three tiny milia on the scar site treated by pneumatic injection (Figure 1d).

The pneumatic jet injector has shown good efficacy in inducing skin remodeling and wound healing [1,3,4]. Pinpoint bleeding, transient erythema and edema and focal tenderness are common and resolve within 24 hours [3]. In our two cases, milia appeared at the injected site an uncommon adverse effect of pneumatic injection treatment.

Milia are very common superficial keratinous cysts, clinically seen as pearly white cone-shaped lesions with a diameter of 1-2 mm. These superficial lesions can be primary or secondary. Secondary causes include trauma such as burns, subepidermal bullae, dermabrasion or ablative laser resurfacing [4]. It is still unclear whether trauma creates milia undifferentiated pilosebaceous cells to proliferate. However 
Citation: Choi SY, Ahn GR, Kim BJ, Kim MN, Hong CK. The Formation of Multiple Milia Induced by Pneumatic Injector Therapy for Scar Remodeling. J Clin Investigat Dermatol. 2017;5(2): 2.

ISSN: 2373-1044

considering that calcified through epidermal implantation or by providing a stimulus for nodules developing after neonatal heel sticks have been well documented, especially in high-risk neonates after repeated skin puncture [5]. We could hypothesize that mechanical stress induced by the pneumatic injector caused micro-trauma to the basement membrane of the skin and resulted in plugging of the pilosebaceous or eccrine sweat ducts, leading to development of multiple milia at the injection site. Since both two cases used same HA material for pneumatic injection treatment the possibility of injected HA promoted the growth of keratinous cyst by its volumizing effect or further activation of cellular signal transduction pathway is still remaining.

We reported our two cases of milia formation after pneumatic injector therapy. Physicians should be aware of the possibility of secondary milia formation after pneumatic injector therapy for scar remodeling. Further clinical experiences or pre-clinical investigations of pneumatic injection treatment using various injection materials are expected.

\section{References}

1. Lee JW, Kim BJ, Kim MN, Lee CK (2010) Treatment of acne scars using subdermal minimal surgery technology. Dermatol Surg 36: 1281-1287.

2. Mitragotri S (2006) Current status and future prospects of needle-free liquid jet injectors. Nat Rev Drug Discov 5: 543-548.

3. Levenberg A, Halachmi S, Arad-Cohen A, Ad-EI D, Cassuto D, et al. (2010) Clinical results of skin remodeling using a novel pneumatic technology. Int J Dermatol 49: 1432-1439.

4. Kim BJ, Yoo KH, Kim MN (2009) Successful treatment of depressed scars of the forehead secondary to herpes zoster using subdermal minimal surgery technology. Dermatol Surg 35: 1439-1440.

5. Berk DR, Bayliss SJ (2008) Milia: A review and classification. J Am Acad Dermatol 59: 1050-1063. 